\title{
Effect of tyrosine kinase inhibitors, imatinib and nilotinib, in murine lipopolysaccharide-induced acute lung injury during neutropenia recovery
}

In Kyoung Kim ${ }^{1 \dagger}$, Chin Kook Rhee ${ }^{1 \dagger}$, Chang Dong Yeo ${ }^{1}$, Hyeon Hui Kang ${ }^{1}$, Dong Gun Lee ${ }^{2}$, Sang Haak Lee ${ }^{1}$ and Jin Woo Kim ${ }^{1 *}$

\begin{abstract}
Introduction: Neutrophil recovery has been implicated in deterioration of oxygenation and exacerbation of preexisting acute lung injury (ALI). The aim of this study was to investigate whether imatinib or nilotinib was effective on lipopolysaccharide (LPS)-induced ALI during neutropenia recovery in mice.

Methods: Mice were rendered neutropenic with cyclophosphamide prior to the intratracheal instillation of LPS. Imatinib or nilotinib was administrated by oral gavage during neutropenia recovery. In order to study the effects of drugs, mice were killed on day 5 and blood, bronchoalveolar lavage (BAL) fluid and lung tissue samples were obtained. The lung wet/dry weight ratio and protein levels in the BAL fluid or lung tissue were determined.

Results: Treatment with imatinib or nilotinib significantly attenuated the LPS-induced pulmonary edema, and this result was supported by the histopathological examination. The concentrations of tumor necrosis factor- $\alpha$, interleukin (IL)-1 $1 \beta, I L-6$ and myeloperoxidase in BAL fluid were significantly inhibited by imatinib or nilotinib in mice of ALI during neutropenia recovery. The mRNA expressions of platelet-derived growth factor receptor $\beta$ and c-KIT in imatinib or nilotinib group were significantly lower than LPS group.

Conclusions: Our data indicated that imatinib or nilotinib effectively attenuated LPS-induced ALI during neutropenia recovery. These results provide evidence for the therapeutic potential of imatinib and nilotinib in ALI during neutropenia recovery.
\end{abstract}

Keywords: Acute lung injury, neutropenia recovery, imatinib, nilotinib, platelet-derived growth factor receptor (PDGFR)

\section{Introduction}

Acute lung injury (ALI) and acute respiratory failure are the major cause of morbidity and the major reason for ICU admission in cancer patients [1-4]. Neutropenia, characterized by low count of neutrophils, which have a critical role in the pathophysiology of acute respiratory distress syndrome (ARDS) and ALI, is mostly a commonly expected event in the numerous cancer patients who are administered chemotherapy $[5,6]$. Neutropenia recovery may be related to an increased risk of deteriorating oxygenation

\footnotetext{
* Correspondence: medkjw@catholic.ac.kr

+ Contributed equally

'Division of Pulmonary and Critical Care Medicine, Department of Internal Medicine, School of Medicine, The Catholic University of Korea, Seoul, Korea Full list of author information is available at the end of the article
}

and may exacerbate pre-existing ALI associated with infectious or noninfectious causes [7-11].

Most clinical studies have focused on the significant role of ALI before neutropenia recovery to detect confounding factors affecting the recovery. However, experimental studies to prevent or attenuate factors for ALI/ARDS after neutropenia recovery have been lacking to date, although ARDS has been widely reported during neutropenia recovery.

Lipopolysaccharide (LPS), a component of gram-negative bacterial endotoxin, is recognized as the main component causing ALI. It has been shown that ALI due to LPS instillation results in an increase in the numbers of total cells and neutrophils, as well as various proimflammatory cytokines such as TNF- $\alpha$, IL-1 $\beta$ and IL- 6 in bronchoalveolar 
lavage (BAL) fluid, and increased protein leakage, pulmonary elastance and resistance. There is also recent clinical evidence that increased TNF- $\alpha$, IL-1 $\beta$ and IL- 6 levels are associated with poor patient outcome in ALI.

Imatinib and nilotinib (Novartis Pharmaceuticals) are protein tyrosine kinase inhibitors whose main targets include platelet-derived growth factor (PDGF) receptor (PDGFR), discoidin domain receptor, stem cell factor receptor (KIT), Abelson kinase (ABL) and the oncogenic breakpoint cluster region-Abelson kinase (BCR-ABL) that causes chronic myeloid leukemia [12]. Imatinib and its advancer, nilotinib, have been shown to possess other beneficial pharmacological effects such as anti-inflammatory activities and antifibrotic effects $[13,14]$. There is evidence that these agents specifically attenuate airway hyper-reactivity [15] and its ability to inhibit PDGFR tyrosine kinase [14]. In a recent case report, PDGF has been known to play a key role in acute lung injury [16]. However, whether imatinib and nilotinib could affect ALI during neutropenia recovery and ultimately improve the ALI is unknown.

We hypothesized that imatinib and nilotinib may inhibit the cytokine production involved in the development of ALI. Therefore, the aim of the present study was to evaluate whether imatinib or nilotinib was effective in LPSinduced ALI during neutropenia recovery in a mouse model and whether these agents suppress the production of proinflammatory cytokines.

\section{Materials and methods}

\section{Animals and treatment}

Female 5-week-old ICR mice, weighing 18 to $22 \mathrm{~g}$ ( $n=10$ per group), were purchased from Orient Bio Experimental Animal Center, Kyoungki, Korea. All animals were maintained in a pathogen-free environment and had access to food and water ad libitum. Mice were randomly allocated into four groups: (i) control; (ii) cyclophosphamide + LPS (2 $\mu \mathrm{g} / \mathrm{g}$, Sigma, St. Louis, MO, USA); (iii) cyclophosphamide + LPS + imatinib (100 mg/kg, twice a day); and (iv) cyclophosphamide + LPS + nilotinib $(100 \mathrm{mg} / \mathrm{kg}$, once a day). Neutropenia was induced in the animals by intraperitoneal injections of cyclophosphamide of $150 \mathrm{mg} / \mathrm{kg}$ on day -5 (before imatinib or nilotinib administration) and $100 \mathrm{mg} / \mathrm{kg}$ on day -2 . Imatinib or nilotinib (provided by Novartis Pharmaceuticals, Basel, Switzerland) was administered by oral gavage on day 0 and continued until euthanasia. In the groups (ii), (iii), and (iv), mice were given LPS $(2 \mu \mathrm{g} / \mathrm{g})$ through intratracheal instillation on day 2 . Mice were sacrificed on day 5 .

We also performed two additional experiments. First, we added two groups (LPS and saline) and compared the degree of lung injury. In the LPS group, mice was given LPS $(2 \mu \mathrm{g} / \mathrm{g})$ through intratracheal instillation without treatment of cyclophosphamide. Instead of LPS, the saline group received the same amount of saline through intratracheal instillation without cyclophosphamide treatment. Second, we gave imatinib or nilotinib after, instead of before, LPS administration. We compared the effect of imatinib or nilotinib in the pre- and post-LPS groups.

The experiments were approved by the ethical committee on animal experiments of The Catholic University of Korea.

\section{Quantification of lung wet/dry weight ratio}

Mice were sacrificed by $\mathrm{CO}_{2}$ asphyxiation. At the completion of the experiment, the upper lobe of the right lung was excised and immediately weighed to determine the wet/dry (W/D) weight ratio. It was then dried at $60^{\circ} \mathrm{C}$ for $72 \mathrm{hrs}$, and reweighed. The ratio of W/D weight was used to quantify lung water content [17].

\section{Lung histopathology}

After sacrifice, the lungs were inflated, fixed in $4 \%$ paraformaldehyde for $24 \mathrm{hrs}$, and then embedded in paraffin wax. Sections were cut at 4- $\mu \mathrm{m}$ thickness using a microtome, and stained with H\&E using standard techniques for histological changes.

\section{Bronchoalveolar lavage}

The trachea was cannulated with a small catheter, and BAL fluid was collected by washing the lungs with $0.8 \mathrm{ml}$ of ice-cold sterile PBS. Total cell counts in BAL fluid were measured using a hemocytometer. Total leukocyte counts were obtained by light microscopic evaluation and the percentages of BAL fluid macrophages, neutrophils, eosinophils and lymphocytes were obtained by counting 500 leukocytes on randomly selected portions. BAL fluid was analyzed by cytospin stained with Diff Quik (Sysmax, Tokyo, Japan). The supernatant of BAL fluid was aliquoted and frozen at $-80^{\circ} \mathrm{C}$ until further analysis.

\section{Myeloperoxidase activity assay}

Myeloperoxidase (MPO) concentration in BAL fluid was determined using the Mouse MPO ELISA kit (Uscn Life Science, Wuhan, China) according to the manufacturer's instructions. Briefly, BAL fluid was centrifuged at 13,000 rpm for 10 minutes at $4^{\circ} \mathrm{C}$ and the supernatant discarded. The leukocyte pellet was suspended again in extraction buffer. The sensitivity of the assay was $0.78 \mathrm{ng} / \mathrm{ml}$. Optical density was measured at $450 \mathrm{~nm}$ by use of a microplate reader.

\section{Enzyme-linked immunosorbent assay}

The concentrations of TNF- $\alpha$, IL- 6 and IL- $1 \beta$ in BAL fluids were measured using an ELISA kit (R\&D Systems, Minneapolis, MN, USA). The sensitivities of the assay were $15.6 \mathrm{pg} / \mathrm{ml}, 3 \mathrm{pg} / \mathrm{ml}$ and $7 \mathrm{pg} / \mathrm{ml}$, respectively. The protocol was performed according to the manufacturer's instructions. 


\section{Western blot}

Separated lung tissues were homogenized in radio immunoprecipitation assay (RIPA) cell lysis buffer $(150 \mathrm{mM}$ $\mathrm{NaCl}, 1 \%$ triton $\mathrm{X}-100,1 \%$ sodium deoxycholate, $0.1 \%$ SDS, $50 \mathrm{mM}$ Tris- $\mathrm{HCl}, \mathrm{pH} 7.5$, and $2 \mathrm{mM}$ ethylenediaminetetraacetic acid (EDTA)) containing a mixture of protease inhibitors (GenDEPOT, CA, USA) and then centrifuged at $13,000 \mathrm{rpm}$ for 15 minutes at $4^{\circ} \mathrm{C}$, followed by collection and storage of supernatant at $-80^{\circ} \mathrm{C}$. Protein samples were separated by $8 \%$ sodium dodecyl sulfate polyacrylamide gel electrophoresis and transferred to Immobilon-P (Millipore) polyvinylidene difluoride membranes. The membrane was blocked with $5 \%$ skimmed milk (Difco/Becton Dickinson, Atlanta, GA, USA) for $2 \mathrm{hrs}$ at room temperature, followed by incubation with a 1:200 dilution of anti-PDGFR- $\beta$ (Santa Cruz Biotechnology, Santa Cruz, CA, USA) or a 1:1,000 dilution anti-pPDGFR- $\beta$ antibody (Cell Signaling, Danvers, MA, USA) overnight at $4^{\circ} \mathrm{C}$. After incubation, the blot was washed with Tris-buffered saline containing $0.1 \%$ Tween 20 (TBS-T) for 30 minutes and incubated with a 1:2,000 dilution of goat anti-rabbit secondary antibody (Santa Cruz Biotechnology, Santa Cruz, CA, USA) for 2 hrs at room temperature. The blot was then washed with TBS-T for $1 \mathrm{hr}$, developed using the ECL Western Blotting Analysis System, and exposed to film.

\section{Real-time polymerase chain reaction}

Total RNA was extracted from lung tissue using the TRIzol reagent ${ }^{\mathrm{TM}}$ (Invitrogen, Carlsbad, CA, USA) according to the manufacturer's recommendations. After extraction and quantification of total RNA, real-time PCR reactions were performed using a QuantiTect SYBR Green RT-PCR kit (Qiagen, Valencia, CA, USA). Each PCR was carried out in a final volume of $25 \mu \mathrm{l}(100 \mathrm{ng} \mathrm{cDNA}, 1 \mu \mathrm{mol} / \mathrm{l}$ of primers, $2 \times$ SYBR RT-PCR Master Mix, RNase-free water). The PCR conditions were $95^{\circ} \mathrm{C}$ (10 minutes), followed by 40 cycles at $95^{\circ} \mathrm{C}(15 \mathrm{sec}), 60^{\circ} \mathrm{C}$ ( 1 minute), and the standard denaturation curve. Primer sequences used were: PDGFR- $\beta$ [NCBI:NM001146268.1], forward 5'TGGGCTTCAGCTACCAAGTG-3', reverse 5' - AAGG TGCTGCCTTTGGAGAT-3'; $\beta$-actin (housekeeping control, [NCBI:NM007393.3]), forward 5'-ACAGGAAGTC CCTTGCCATC-3', reverse 5'-AGGGAGACCAAAAGC CTTCA-3'. Gene expression was quantified using standard curves for the respective cDNA products. To normalize the content of cDNA samples, the comparative threshold (CT) cycle method, consisting of the normalization of the number of target gene copies versus the housekeeping gene $\beta$-actin, was used. The changes in target signal were expressed as:

$$
\Delta \Delta \mathrm{C}_{\mathrm{T}}=\Delta \mathrm{C}_{\mathrm{T}, \text { treatment }}-\Delta \mathrm{C}_{\mathrm{T}, \text { control }}
$$

Relative changes were calculated as $2^{-\Delta \Delta C T}$.

\section{Statistical analysis}

The data were analyzed by one-way analysis of variance (ANOVA) followed by Dunnett's multiple range test, using Graph-Pad Prism version 5.00 for Windows (GraphPad Software, San Diego, CA, USA). All data are expressed as means $\pm \mathrm{SD}$, and a $P$-value $<0.05$ was accepted as statistically significant. SPSS for Windows was used for the statistical tests.

\section{Results}

\section{Effect of cyclophosphamide on neutrophils}

The cytotoxic effect of cyclophosphamide in mice was monitored by neutrophil counts. In mice given cyclophosphamide, neutrophils in the peripheral blood reached a minimum at day 1 and recovered at day 5 (Figure 1).

\section{Effect of imatinib or nilotinib on lung histopathology}

To evaluate the effect of imatinib or nilotinib in the histopathological changes in the lung in LPS-induced ALI, lung tissues were assessed after the administration of LPS during neutropenia recovery with or without treatment. We observed marked acute alveolar damage, acute inflammation and interstitial edema in LPSinduced ALI during neutropenia recovery (Figure 2B), compared with the control group (Figure 2A). In the group pretreated with imatinib or nilotinib before LPS, administration of drugs effectively reduced the inflammatory changes in the lung (Figure 2C and 2D).

\section{Effect of imatinib or nilotinib on pulmonary edema}

Pulmonary edema formation, indicative of pulmonary vascular leakage, happens with an increase in the lung W/D ratio. To investigate the effect of imatinib or nilotinib on LPS-induced lung edema, the lung W/D ratios and the concentration of albumin in BAL fluids were measured. As shown in Figure 3A, we found that there was an obvious increase in W/D ratio in the group that had LPS administration during neutropenia recovery, compared with the control group. However, administration of imatinib or nilotinib prior to LPS significantly reduced the lung W/D ratios. The level of albumin in BAL fluid was increased in mice challenged with LPS during neutropenia recovery compared with control mice, whereas treatment with imatinib or nilotinib prior to LPS significantly attenuated the albumin level (Figure 3B).

\section{Effect of imatinib or nilotinib on inflammatory cells}

To investigate the protective effect of imatinib or nilotinib on LPS-induced ALI during neutropenia recovery, we detected the total inflammatory cell counts in BAL 


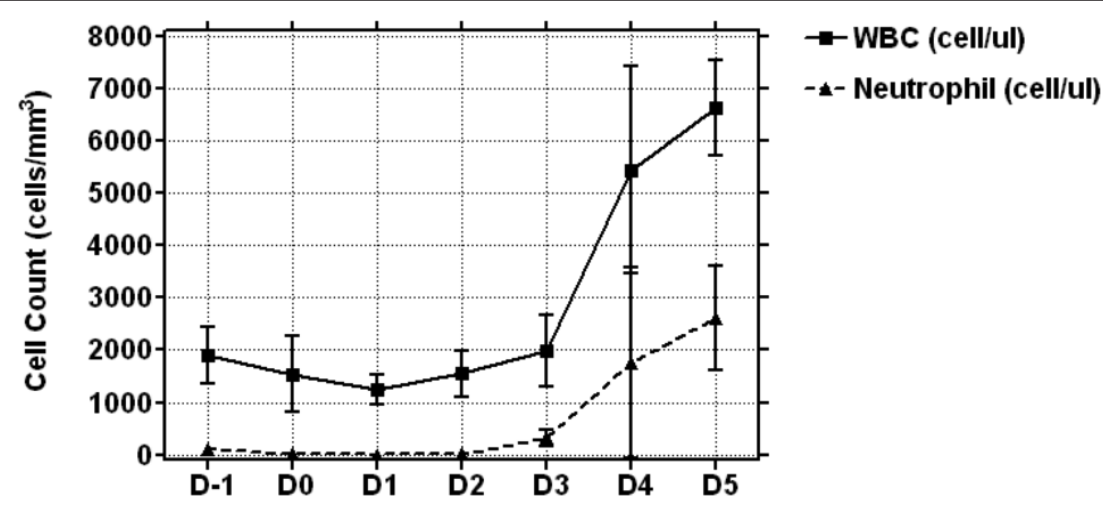

Figure 1 Effect of cyclophosphamide in mice. All mice received $150 \mathrm{mg} / \mathrm{kg}$ cyclophosphamide by intraperitoneal injection on day (D) -5 before they became neutropenic and $100 \mathrm{mg} / \mathrm{kg}$ on day -2 before administration of imatinib or nilotinib. Neutrophils were measured in the peripheral blood every day to monitor the cytotoxic effect of cyclophosphamide until mice were sacrificed. WBC, white blood cell.

fluid from mice treated with LPS with or without imatinib or nilotinib. As shown in table 1, inflammatory cells and neutrophils in the BAL fluid were significantly increased after administration of LPS during neutropenia recovery $(P<0.01)$, whereas we observed that administration of imatinib or nilotinib significantly reduced the number of total cells and neutrophils compared with the LPS group $(P<0.01)$.

\section{Effect of imatinib or nilotinib on inflammatory cytokines and MPO}

To assess the anti-inflammatory effect of imatinib or nilotinib, we analyzed the proinflammatory cytokines, including TNF- $\alpha$, IL-6, IL-1ß, and MPO in BAL fluid. We found that the concentrations of TNF- $\alpha$, IL-6, IL$1 ß$, and MPO were dramatically increased in BAL fluid after LPS administration during neutropenia recovery. The concentrations of TNF- $\alpha$, IL- 6 and IL- $1 \beta$ in response to LPS were $812.16 \pm 84.88 \mathrm{pg} / \mathrm{ml}, 394.19 \pm$ $67.00 \mathrm{pg} / \mathrm{ml}$ and $1,476.81 \pm 268.95 \mathrm{pg} / \mathrm{ml}$, respectively, indicating approximately 58.2-, 109.0- and 8.8-fold increases compared with control group. In contrast, administration of imatinib or nilotinib after LPS challenge effectively decreased the levels of TNF- $\alpha$, IL-6, IL$1 ß$ and MPO (Figure 4).

\section{Effect of imatinib or nilotinib on activation of PDGFR-}

Western blot analysis of the phosphorylation of PDGFR$ß$ revealed significant upregulation in the group with LPS during neutropenia recovery, whereas the group treated with imatinib or nilotinib there was a reduction toward control levels (Figure 5). There was no significant difference in the expression of non-phosphorylated PDGFR- $\beta$ in mice exposed to LPS during neutropenia recovery compared with the control group.

\section{Effect of imatinib or nilotinib on mRNA expression of PDGFR-ß}

Real-time PCR was used to analyze the effect of imatinib or nilotinib on the mRNA expression of PDGFR- $ß$ in

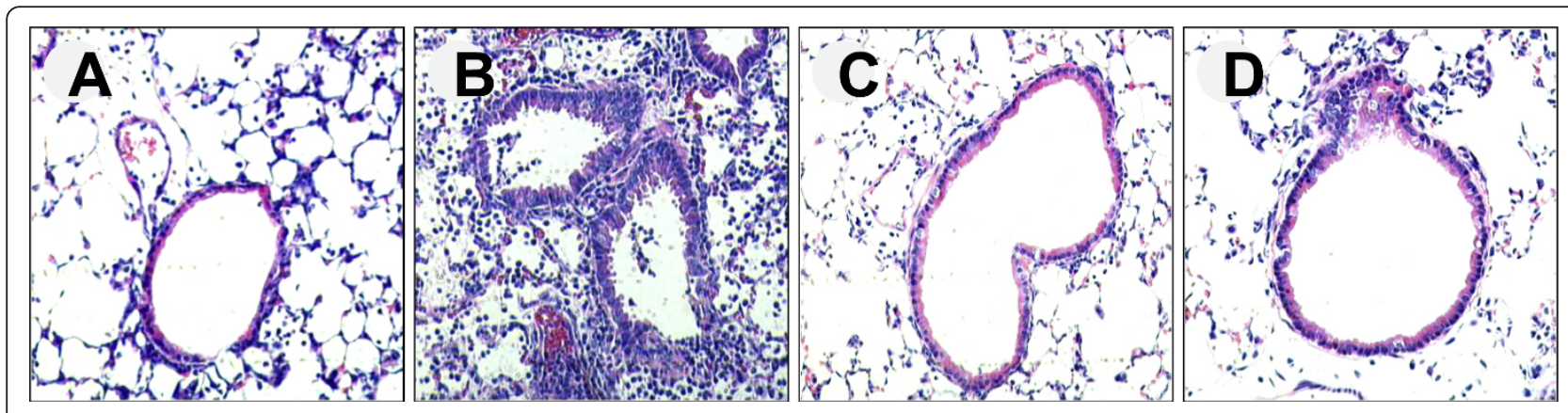

Figure 2 Effect of imatinib or nilotinib on the histopathological changes in the lung in mice lipopolysaccharide-induced acute lung inury. Representative images of H\&E-stained lung sections from four experimental groups: (A) control group; the lung structure is normal, (B) cyclophosphamide (C) + lipopolysaccharide (LPS) group; LPS induced acute lung damage with interstitial edema, hemorrhage, thickening of the alveolar wall and infiltration of inflammatory cells into the interstitium and alveolar spaces, (C) C + LPS + imatinib (I) group, (D) C + LPS + nilotinib (N) group; lung injury was attenuated by treatment with imatinib or nilotinib. 

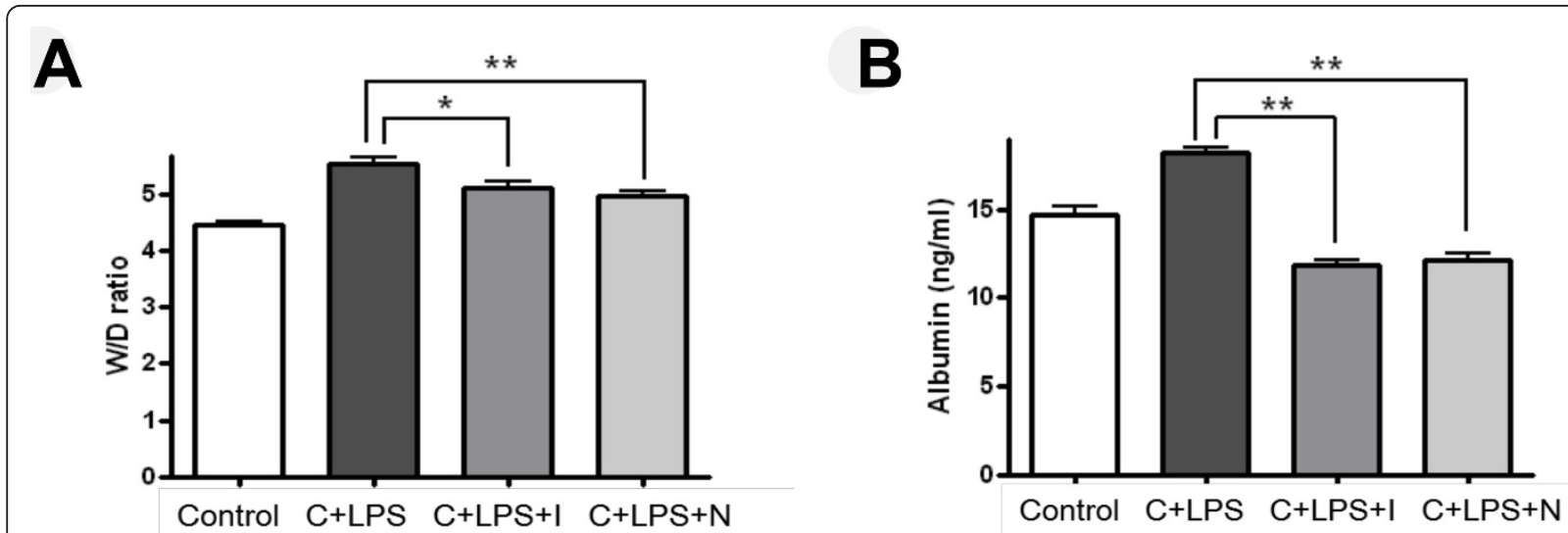

Figure 3 Effect of imatinib or nilotinib on lung edema in mice with lipoplysaccharide-induced acute lung injury. After lipopolysaccharide (LPS) administration, treatment with imatinib or nilotinib markedly decreased (A) lung wet/dry (W/D) weight ratio and (B) albumin. The lower lobe of the right lung was removed and weighed (wet weight), dried at $60^{\circ} \mathrm{C}$ for $72 \mathrm{hrs}$, and weighed again (dry weight). The W/D ratio was used as an indicator of lung edema formation. C, cyclophosphamide; I, imatinib; N, nilotinib. Asterisks indicates significant differences to the $C+$ LPS group $\left({ }^{*} P<0.05,{ }^{* *} P<0.01\right)$.

lung tissue after the LPS challenge. As shown in Figure 6, the administration of LPS during neutropenia recovery induced a significant increase in the mRNA expression of PDGFR- $\beta$ as compared to the control group. Imatinib or nilotinib significantly reduced LPS-induced PDGFR- $\beta$ expression (Figure $6, P<0.01$ ).

\section{Comparison of degree of lung injury}

We compared the degree of lung injury in the LPS group and the cyclophosphamide + LPS group. We also compared the control and the saline group. In the histopathology, there was little inflammation in the control or the saline group. Acute lung damage with interstitial edema, hemorrhage, thickening of the alveolar wall and infiltration of inflammatory cells into the interstitium and alveolar spaces were observed in both the LPS and the cyclophosphamide + LPS group (Figure 7A). In BAL cell count analysis, there was a significant difference between the control and the saline group in the number of total cells $(P<0.05)$. However, the neutrophil count did not differ significantly between the control and the saline group. There was also significant difference between the LPS group and the cyclophosphamide + LPS group in the number of total cells $(P<0.01)$.
However, the neutrophil count did not differ significantly in the LPS group and the cyclophosphamide + LPS group (Figure 7B). In the analysis of MPO, there was no significant difference between the control and the saline group. There was also no significant difference between the LPS group and the cyclophosphamide + LPS group (Figure 7C).

\section{Post-treatment effect of imatinib or nilotinib}

In the histopathology, imatinib or nilotinib given pre- or post-induction of lung injury attenuated lung injury compared with cyclophosphamide + LPS (Figure 8A). Compared with the cyclophosphamide + LPS group, the total cell count did not differ significantly in the group that received imatinib post treatment. However, the neutrophil count differed significantly in the group receiving imatinib post treatment $(P<0.05)$. Compared with cyclophosphamide + LPS group, both total cell and neutrophil count were significantly lower in post-treatment of nilotinib group $(P<0.01)$. The total cell and neutrophil counts were significantly higher in post-treatment imatinib group compared with the pre-treatment group $(P<0.01)$. However, there was no significant difference between the pre- and post-treatment nilotinib group in the numbers of total

Table 1 Results of bronchiolar lavage fluid analysis $\left(10^{4} / \mathrm{ml}\right)$ in the four groups

\begin{tabular}{lllll}
\hline & Control & C + LPS & C + LPS + I & C + LPS + N \\
\hline Total cells & $6.00 \pm 2.35$ & $80.60 \pm 16.30^{* *}$ & $36.20 \pm 7.26^{\# \#}$ & $43.40 \pm 6.91^{\# \#}$ \\
Macrophages & $3.67 \pm 2.15$ & $4.18 \pm 0.68$ & $5.63 \pm 3.20$ & $3.49 \pm 4.88$ \\
Lymphocytes & $0.00 \pm 0.00$ & $0.34 \pm 0.56$ & $0.00 \pm 0.00$ & $0.00 \pm 0.00$ \\
Neutrophils & $0.00 \pm 0.00$ & $71.46 \pm 16.09^{* *}$ & $26.38 \pm 2.85^{\# \#}$ & $14.46 \pm 20.24^{\# \#}$ \\
Eosinophils & $0.34 \pm 0.21$ & $4.64 \pm 3.95$ & $4.20 \pm 4.81$ & $2.04 \pm 2.83$ \\
\hline
\end{tabular}

Results are presented as mean \pm SD. LPS, lipopolysaccharide; $C$, cyclophosphamide; l, imatinib; $N$, nilotinib. ${ }^{* *} P<0.01$ for control versus $C+$ LPS; ${ }^{\# \# ~}<0.01$ for $C+$ LPS versus $\mathrm{C}+\mathrm{LPS}+\mathrm{I}$ or $\mathrm{N}$. 


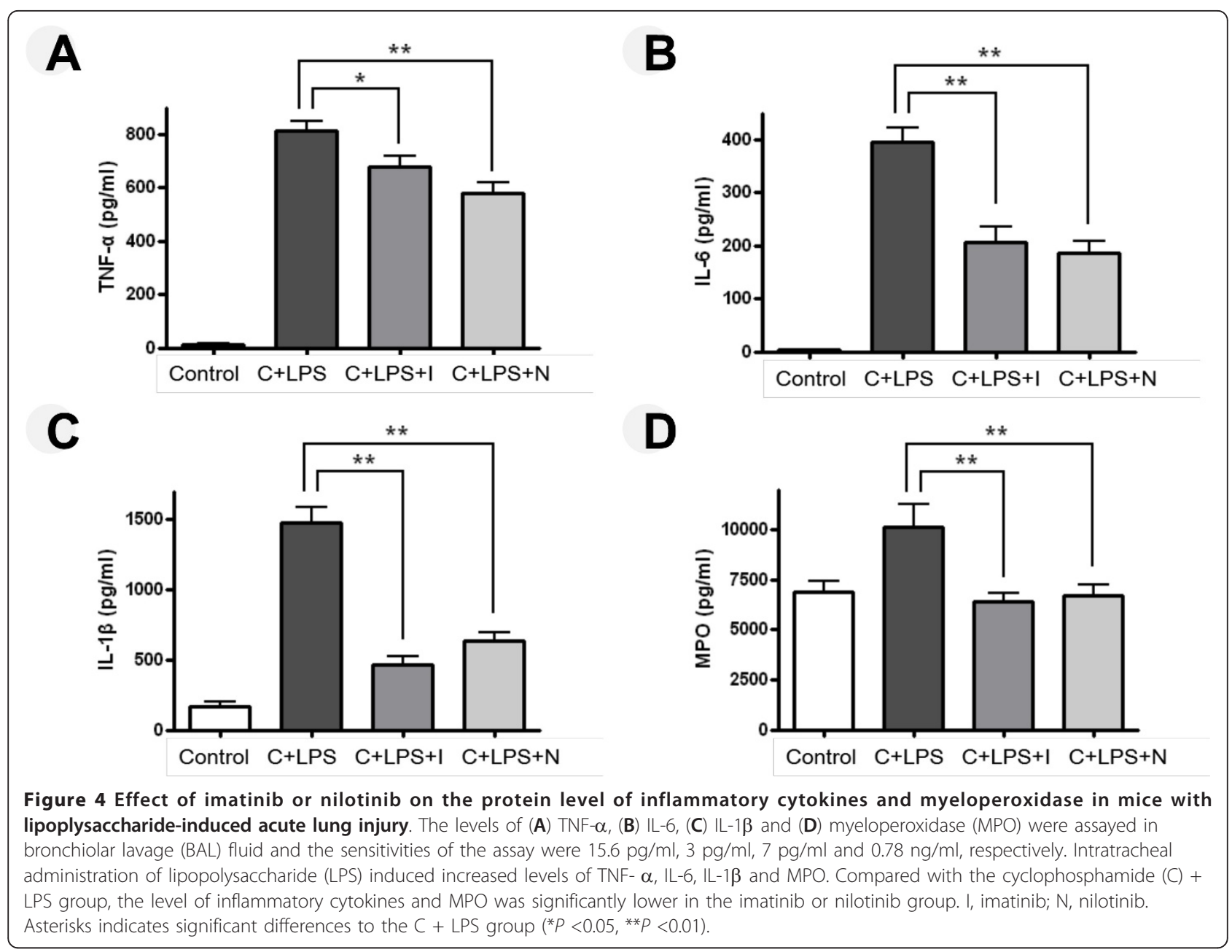

cells and neutrophils (Figure 8B). Compared with the cyclophosphamide + LPS group, the albumin level was significantly lower in the imatinib and nilotinib post-treatment groups $(P<0.01)$. Compared with imatinib and nilotinib post-treatment groups, the albumin level was also significantly lower in the imatinib and nilotinib pre- treatment groups (Figure $8 \mathrm{C}, \mathrm{P}<0.01$ ). Compared with the cyclophosphamide + LPS group, MPO was significantly lower in the imatinib and nilotinib post-treatment groups $(P<0.05)$. There was no significant difference in MPO between the imatinib or nilotinib pre- and post-treatment groups (Figure 8D).

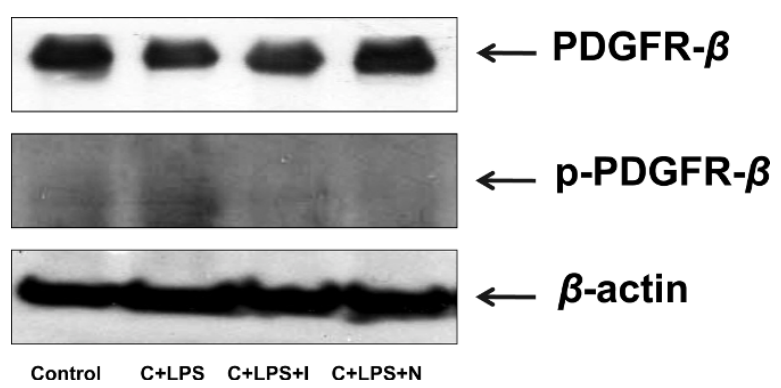

Figure 5 Effect of imatinib or nilotinib on activation of p-platelet-derived growth factor receptor- $B$ in mice with lipoplysaccharideinduced acute lung injury. Lung tissues were homogenized and the extracted protein samples were separated by SDS-PAGE using 8\% PAGE gel, followed by transfer to $0.45 \mu \mathrm{m}$ polyvinylidene fluoride membranes. The membranes were blocked with $5 \%$ skimmed milk and exposed overnight at $4^{\circ} \mathrm{C}$ to specific anti-platelet-derived growth factor receptor- $\beta$ (PDGFR- $\beta$ ) and anti-phospho-PDGFR- $\beta$ antibodies. LPS, lipopolysaccharide; C, cyclophosphamide; I, imatinib; N, nilotinib. 


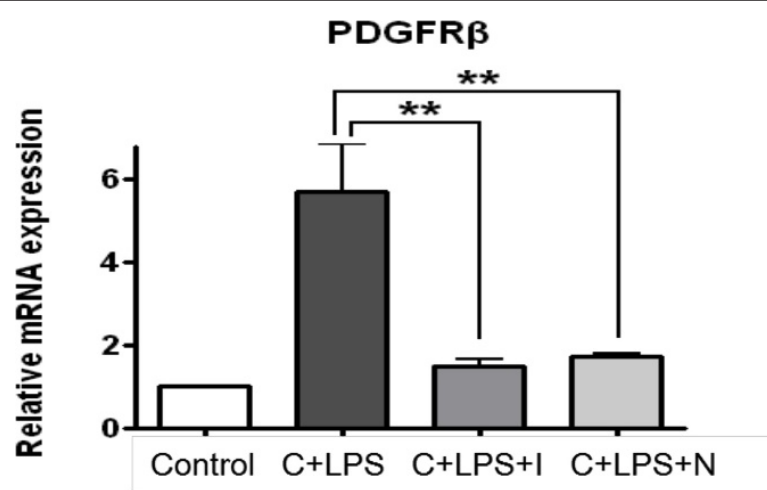

Figure 6 Effect of imatinib or nilotinib treatment on mRNA expression of platelet-derived growth factor receptor in mice with lipoplysaccharide-induced acute lung injury. The mRNA expression of platelet-derived growth factor receptor- $\beta$ (PDGFR) was measured in lung tissues. In real-time PCR analysis, to normalize the content of CDNA samples, the comparative threshold (CT) cycle method, consisting of the normalization of the number of target gene copies versus the housekeeping gene $\beta$-actin, was used. LPS, lipopolysaccharide; C, cyclophosphamide; I, imatinib; N, nilotinib. Asterisks indicate significant differences from the C + LPS group ( $\left.{ }^{* *} P<0.01\right)$.

\section{Discussion}

In this study, we observed the pretreatment effect of imatinib and nilotinib in LPS-induced lung injury in the neutropenic mouse model. Although many cases of ALI/ARDS during neutropenia recovery have been reported [18], risk factors or marked therapeutic agents in patients experiencing neutropenia recovery have not previously been studied.

Chemotherapy is administered to the majority of cancer patients, with neutropenia being a frequent adverse event. In patients, pulmonary infiltrates develop during neutropenia and considerably worsen upon neutropenia

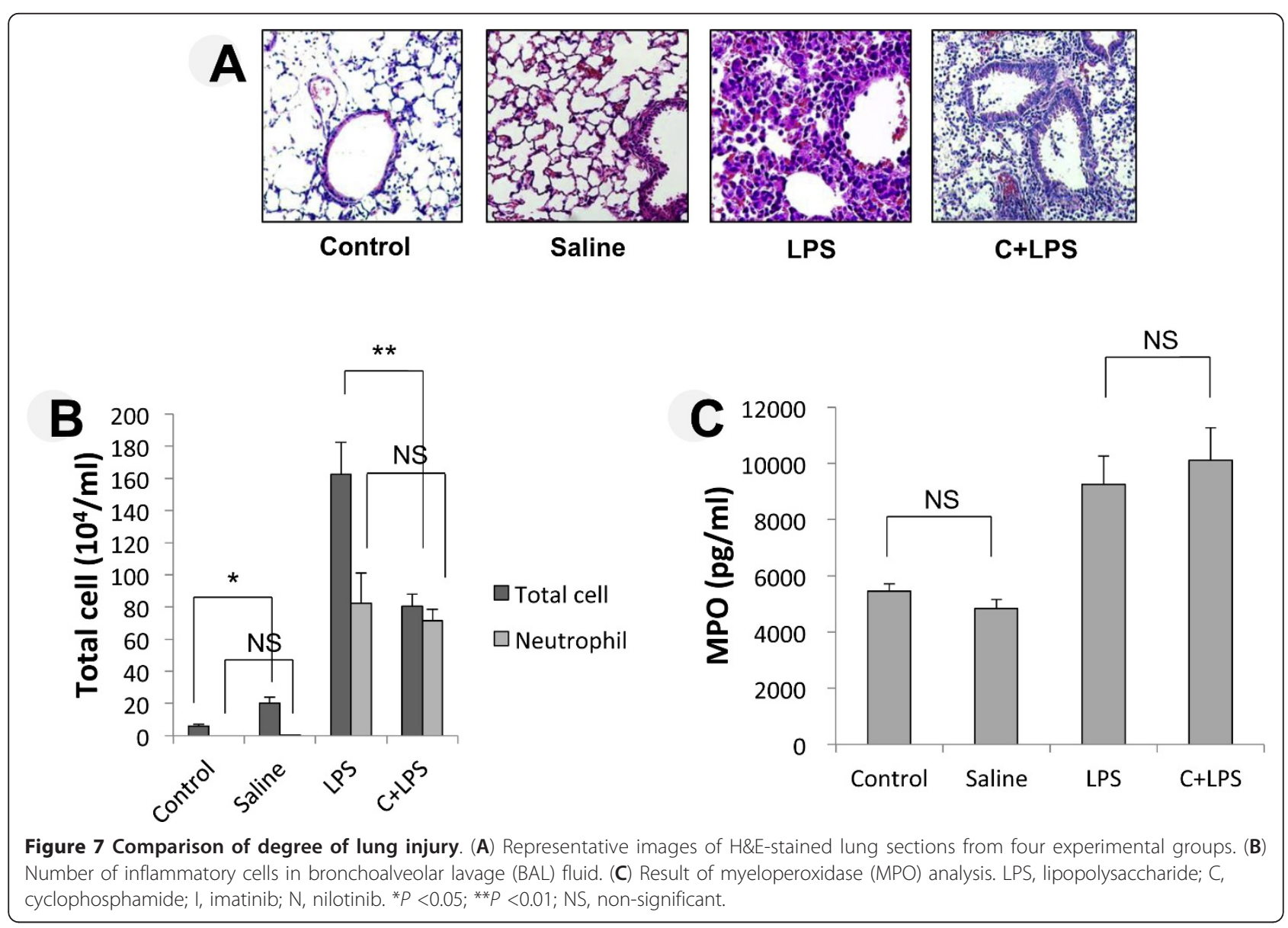




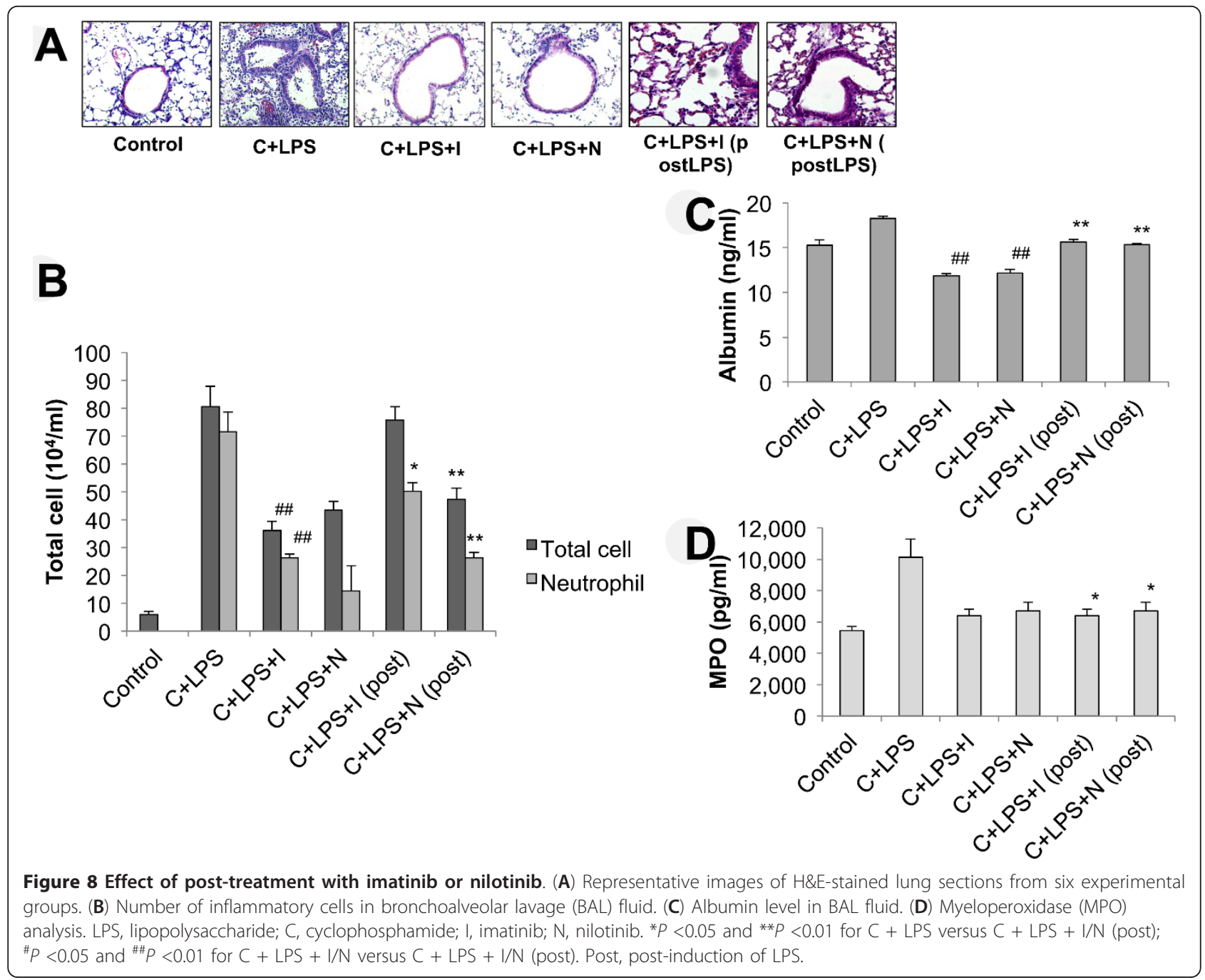

recovery [10]. A few case reports have been published showing that neutropenia recovery carries a risk of acute respiratory failure such as ALI and ARDS $[19,20]$. Azoulay et al. [20] showed that cancer patients recovering from neutropenia were frequently admitted for acute respiratory failure and that one third of patients experienced ARDS during neutropenia recovery. During neutropenia recovery, the accumulation of activated neutrophils in the pulmonary vasculature generally occurred [6]. Activated neutrophils that quickly accumulate in the pulmonary parenchyma following endotoxin administration [21] or hypovolemic shock [22] have a major role in the development of inflammatory response in ALI [23]. Inflammatory cells, including neutrophils and lymphocytes, in BAL fluid have a principal role in the pathogenesis of ALI [24].

PDGF, one of the growth factors whose receptors are targeted by imatinib and nilotinib, plays a key role in the pathogenesis of lung diseases including pulmonary fibrosis, ALI and ARDS [17,25]. PDGF is a chemotactic factor for monocytes and granulocytes during inflammation and overexpression of PDGF can induce inflammatory injury [26]. Previous studies suggest that PDGF may be an important factor in ALI. Snyder et al. [27] showed that the concentration of PDGF is significantly higher in patients with ALI than in normal or control patients. Budinger et al. [28] showed that there was a significant negative correlation between active TGF- $\beta 1$ (stimulator of PDGF) levels and ventilator-free days and ICU-free days. In addition to these clinical data, Walsh et al. [29] showed that PDGF plays a crucial role in the tissue repair processes of bleomycin-induced lung injury in a rat model and leads to fibroblast proliferation and chemotaxis. There are also many reports that on PDGF as a therapeutic target in ALI. In a previous study, we showed that imatinib and nilotinib attenuated bleomycin-induced ALI by blocking the PDGF pathway [30]. Zhao et al. [31] observed therapeutic effects of bone 
marrow-derived mesenchymal stem cells in lung injury and this effect was accompanied by a decreased in PDGF. Yi and colleagues [32] showed that keratinocyte growth factor can attenuate bleomycin-induced lung injury by decreasing the expression of PDGF.

Recent evidence showed that imatinib and nilotinib have various effects in airway hyper-reactivity and inflammatory responses [30,33-35] as they specifically inhibit PDGFR tyrosine kinase $[14,16,17]$. In this study, we found that treatment of imatinib or nilotinib significantly decreased the phosphorylation of PDGFR-ß. We also confirmed that imatinib or nilotinib could decrease the mRNA expression of PDGFR- $\beta$. These previous reports together with our results suggest that inhibition of PDGF/PDGFR may be a potential approach to prevent lung injury.

We have observed that imatinib and nilotinib have an anti-inflammatory effect. This beneficial effect could contribute to attenuation of lung injury. In BAL fluid analysis, total cells and neutrophils in the BAL fluid were significantly decreased by treatment with imatinib or nilotinib in mice with LPS-induced ALI during neutropenia recovery. Similarly, MPO activity, which indicates neutrophil and macrophage leakage, was significantly increased in BAL fluid after the administration of LPS. These results confirm that a potent protective effect of imatinib or nilotinib on LPS-induced ALI during neutropenia recovery is related to an attenuation of lung inflammation and tissue neutrophilia. The antiinflammatory effect of imatinib and nilotinib is consistent with previous reports. Miyachi et al. [36] and Eklund et al. $[37,38]$ reported that imatinib has an antiinflammatory effect and this drug can be used for the treatment of rheumatoid arthritis. In animal studies previous experiments consistently showed that imatinib and nilotinib decreased total cells and/or neutrophils in BAL fluid $[15,30,33]$.

It has been shown that the early release of several inflammatory and chemotactic cytokines, such as TNF$\alpha$, IL- 6 and IL-1ß, enlarge and facilitate inflammatory responses in ALI $[39,40]$. These are the primary multifunctional cytokines produced from inflammatory cells that exacerbate the extent of lung injury [41]. As in previous studies $[42,43]$, our results showed that the expressions of TNF- $\alpha$, IL- 6 and IL-1 1 were significantly increased in BAL fluid after LPS administration as compared to control group, whereas administration of imatinib or nilotinib significantly downregulated the expression of these cytokines as compared to the LPS group.

\section{Conclusions}

In the present study, we found that administration of imatinib or nilotinib effectively attenuated the LPS- induced ALI during neutropenia recovery in mice. These effects of imatinib or nilotinib were associated with the inhibition of infiltration of inflammatory cells into the lung and reduction of the activation of inflammatory cytokines. Moreover, the beneficial effects of imatinib and nilotinib were closely connected with inhibition of the PDGF pathways, because the PDGF signaling pathway has been involved in developing organ fibrosis and inflammation [44]. However, the accurate intracellular mechanism of the effects of imatinib and nilotinib in mice with LPS-induced ALI still remains to be elucidated. Furthermore, it is necessary to confirm these results in more clinically relevant models.

\section{Key messages}

- Pretreatment with imatinib or nilotinib before LPS exposure during neutropenia recovery, effectively prevented the degree of pulmonary edema and reduced the inflammatory changes in lung tissues.

- Administration of imatinib or nilotinib before instillation of LPS during neutropenia recovery significantly downregulated several inflammatory and chemotactic cytokines, such as TNF- $\alpha$, IL- 6 and IL- $1 ß$.

- Pretreatment with imatinib or nilotinib before instillation of LPS during neutropenia recovery, significantly decreased the phosphorylation of PDGFR- $\beta$ as well as the mRNA expression.

\section{Abbreviations}

ABL: Abelson kinase; ALI: acute lung injury; ANOVA: analysis of variance; ARDS: acute respiratory distress syndrome; BAL: bronchoalveolar lavage; BCR$A B L$ : breakpoint cluster region-Abelson kinase; $C T$ : comparative threshold; EDTA: ethylenediaminetetraacetic acid; ELISA: enzyme-linked immunosorbent assay; H\&E: hematoxylin and eosin; IL: interleukin; KIT: stem cell factor receptor; LPS: lipopolysaccharide; MPO: myeloperoxidase; PBS: phosphatebuffered saline; PCR: polymerase chain reaction;PDGF: platelet-derived growth factor; PDGFR: platelet-derived growth factor receptor; RIPA: radio immunoprecipitation assay; TBS-T: Tris-buffered saline containing $0.1 \%$ Tween 20; TNF: tumor necrosis factor; W/D: wet/dry.

\section{Competing interests}

The authors declare that they have no competing interests.

\section{Authors' contributions}

CKR and JWK designed this study. IKK participated in the animal experiment. IKK, CKR and JWK analyzed the data. DGL contributed to the neutropenia model. IKK, CKR, CDY, HHK, SHL and JWK drafted the manuscript. All authors read and approved the final manuscript.

\section{Acknowledgements}

The authors wish to acknowledge the financial support of the Catholic Medical Center Research Foundation made in the program year of 2012. We thank Novartis Pharmaceuticals (Basel, Switzerland) for donating the raw drug material for the present study. Novartis Pharmaceuticals played no role in the study design, or the collection, analysis, or interpretation of the data, in the writing of the report, or in the decision to submit the paper for publication.

\section{Authors' details}

'Division of Pulmonary and Critical Care Medicine, Department of Internal Medicine, School of Medicine, The Catholic University of Korea, Seoul, Korea. 
2Division of Infectious Diseases, Department of Internal Medicine, School of Medicine, The Catholic University of Korea, Seoul, Korea.

Received: 20 December 2012 Revised: 14 March 2013

Accepted: 20 June 2013 Published: 20 June 2013

\section{References}

1. Kress JP, Christenson J, Pohlman AS, Linkin DR, Hall JB: Outcomes of critically ill cancer patients in a university hospital setting. Am J Respir Crit Care Med 1999, 160:1957-1961.

2. Ewig S, Torres A, Riquelme R, El-Ebiary M, Rovira M, Carreras E, Rano A Xaubet A: Pulmonary complications in patients with haematological malignancies treated at a respiratory ICU. Eur Respir J 1998, 12:116-122.

3. Blot F, Guiguet M, Nitenberg G, Leclercq B, Gachot B, Escudier B: Prognostic factors for neutropenic patients in an intensive care unit: respective roles of underlying malignancies and acute organ failures. Eur J Cancer 1997, 33:1031-1037.

4. Azoulay E, Recher C, Alberti C, Soufir L, Leleu G, Le Gall JR, Fermand JP, Schlemmer B: Changing use of intensive care for hematological patients: the example of multiple myeloma. Intensive Care Med 1999, 25:1395-1401.

5. Coleman MP, Quaresma M, Berrino F, Lutz JM, De Angelis R, Capocaccia R, Baili P, Rachet B, Gatta G, Hakulinen T, Micheli A, Sant M, Weir HK, Elwood JM, Tsukuma H, Koifman S, GA ES, Francisci S, Santaquilani M, Verdecchia A, Storm HH, Young JL: Cancer survival in five continents: a worldwide population-based study (CONCORD). Lancet Oncol 2008, 9:730-756.

6. Rhee CK, Kang JY, Kim YH, Kim JW, Yoon HK, Kim SC, Kwon SS, Kim YK, Kim KH, Moon HS, Park SH, Kim HJ, Lee S, Song JS: Risk factors for acute respiratory distress syndrome during neutropenia recovery in patients with hematologic malignancies. Crit Care 2009, 13:R173.

7. Rinaldo JE, Borovetz $\mathrm{H}$ : Deterioration of oxygenation and abnormal lung microvascular permeability during resolution of leukopenia in patients with diffuse lung injury. Am Rev Respir Dis 1985, 131:579-583.

8. Okubo Y, Nakazawa K: [Recombinant G-CSF and the interstitial pneumonia during MACOP-B therapy in two cases of non-Hodgkin's lymphoma]. Rinsho Ketsueki 1993, 34:473-477.

9. Demuynck H, Zachee P, Verhoef GE, Schetz M, Van den Berghe G, Lauwers P, Boogaerts MA: Risks of rhG-CSF treatment in drug-induced agranulocytosis. Ann Hematol 1995, 70:143-147.

10. Lisukov IA, Kriuchkova IV, Kulagin AD, Gilevich AV, Ostanin AA, Shevela E, Chernykh ER: [The adult respiratory distress syndrome during the recovery of the neutrophil level after autologous bone marrow transplantation]. Ter Arkh 1998, 70:78-79.

11. Todeschini G, Murari C, Bonesi R, Pizzolo G, Verlato G, Tecchio C, Meneghini V, Franchini M, Giuffrida C, Perona G, Bellavite P: Invasive aspergillosis in neutropenic patients: rapid neutrophil recovery is a risk factor for severe pulmonary complications. Eur J Clin Invest 1999, 29:453-457.

12. Manley PW, Stiefl N, Cowan-Jacob SW, Kaufman S, Mestan J, Wartmann M, Wiesmann M, Woodman R, Gallagher N: Structural resemblances and comparisons of the relative pharmacological properties of imatinib and nilotinib. Bioorg Med Chem 2010, 18:6977-6986.

13. Day E, Waters B, Spiegel K, Alnadaf T, Manley PW, Buchdunger E, Walker C, Jarai G: Inhibition of collagen-induced discoidin domain receptor 1 and 2 activation by imatinib, nilotinib and dasatinib. Eur J Pharmacol 2008, 599:44-53.

14. Akhmetshina A, Dees C, Pileckyte M, Maurer B, Axmann R, Jungel A, Zwerina J, Gay S, Schett G, Distler O, Distler JH: Dual inhibition of c-abl and PDGF receptor signaling by dasatinib and nilotinib for the treatment of dermal fibrosis. FASEB J 2008, 22:2214-2222.

15. Berlin AA, Lukacs NW: Treatment of cockroach allergen asthma model with imatinib attenuates airway responses. Am J Respir Crit Care Med 2005, 171:35-39.

16. Loong HH, Yeo W: Imatinib-induced interstitial lung disease and sunitinib-associated intra-tumour haemorrhage. Hong Kong Med J 2008, 14:495-498.

17. Staub NC: Pulmonary edema. Physiol Rev 1974, 54:678-811.

18. Maunder RJ, Hackman RC, Riff E, Albert RK, Springmeyer SC: Occurrence of the adult respiratory distress syndrome in neutropenic patients. Am Rev Respir Dis 1986, 133:313-316.
19. Laufe MD, Simon RH, Flint A, Keller JB: Adult respiratory distress syndrome in neutropenic patients. Am J Med 1986, 80:1022-1026.

20. Azoulay E, Darmon M, Delclaux C, Fieux F, Bornstain C, Moreau D, Attalah H, Le Gall JR, Schlemmer B: Deterioration of previous acute lung injury during neutropenia recovery. Crit Care Med 2002, 30:781-786.

21. Parsey MV, Tuder RM, Abraham E: Neutrophils are major contributors to intraparenchymal lung IL-1 beta expression after hemorrhage and endotoxemia. J Immunol 1998, 160:1007-1013.

22. Abraham E, Bursten $S$, Shenkar R, Allbee J, Tuder R, Woodson P, Guidot DM, Rice G, Singer JW, Repine JE: Phosphatidic acid signaling mediates lung cytokine expression and lung inflammatory injury after hemorrhage in mice. J Exp Med 1995, 181:569-575.

23. Reutershan J, Basit A, Galkina EV, Ley K: Sequential recruitment of neutrophils into lung and bronchoalveolar lavage fluid in LPS-induced acute lung injury. Am J Physiol Lung Cell Mol Physiol 2005, 289:L807-815.

24. Abraham E: Neutrophils and acute lung injury. Crit Care Med 2003, 31 S195-199.

25. Heldin $\mathrm{CH}$, Westermark B: Mechanism of action and in vivo role of platelet-derived growth factor. Physiol Rev 1999, 79:1283-1316.

26. Siegbahn A, Hammacher A, Westermark B, Heldin CH: Differential effects of the various isoforms of platelet-derived growth factor on chemotaxis of fibroblasts, monocytes, and granulocytes. J Clin Invest 1990, 85:916-920

27. Snyder LS, Hertz MI, Peterson MS, Harmon KR, Marinelli WA, Henke CA, Greenheck JR, Chen B, Bitterman PB: Acute lung injury. Pathogenesis of intraalveolar fibrosis. J Clin Invest 1991, 88:663-673.

28. Budinger GR, Chandel NS, Donnelly HK, Eisenbart J, Oberoi M, Jain M: Active transforming growth factor-beta1 activates the procollagen I promoter in patients with acute lung injury. Intensive Care Med 2005, 31:121-128.

29. Walsh J, Absher M, Kelley J: Variable expression of platelet-derived growth factor family proteins in acute lung injury. Am J Respir Cell Mol Biol 1993, 9:637-644.

30. Rhee CK, Lee SH, Yoon HK, Kim SC, Lee SY, Kwon SS, Kim YK, Kim KH, Kim TJ, Kim JW: Effect of nilotinib on bleomycin-induced acute lung injury and pulmonary fibrosis in mice. Respiration 2011, 82:273-287.

31. Zhao F, Zhang YF, Liu YG, Zhou JJ, Li ZK, Wu CG, Qi HW: Therapeutic effects of bone marrow-derived mesenchymal stem cells engraftment on bleomycin-induced lung injury in rats. Transplantation proceedings 2008, 40:1700-1705.

32. Yi ES, Salgado M, Williams S, Kim SJ, Masliah E, Yin S, Ulich TR: Keratinocyte growth factor decreases pulmonary edema, transforming growth factorbeta and platelet-derived growth factor-BB expression, and alveolar type II cell loss in bleomycin-induced lung injury. Inflammation 1998, 22:315-325.

33. Rhee CK, Kim JW, Park CK, Kim JS, Kang JY, Kim SJ, Kim SC, Kwon SS, Kim YK, Park SH, Lee SY: Effect of imatinib on airway smooth muscle thickening in a murine model of chronic asthma. Int Arch Allergy Immunol 2011, 155:243-251

34. Kobayashi M, Kubota T, Uemura Y, Taguchi H: A case of hypereosinophilic syndrome presenting with chronic cough successfully treated with imatinib. Respirology 2009, 14:302-304.

35. El-Agamy DS: Nilotinib ameliorates lipopolysaccharide-induced acute lung injury in rats. Toxicol Appl Pharmacol 2011, 253:153-160.

36. Miyachi K, Ihara A, Hankins RW, Murai R, Maehiro S, Miyashita H: Efficacy of imatinib mesylate (STI571) treatment for a patient with rheumatoid arthritis developing chronic myelogenous leukemia. Clinical rheumatology 2003, 22:329-332.

37. Eklund KK, Lindstedt K, Sandler C, Kovanen PT, Laasonen L, Juurikivi A Wolff H, Mykkanen M, Joensuu H: Maintained efficacy of the tyrosine kinase inhibitor imatinib mesylate in a patient with rheumatoid arthritis. J Clin Rheumatol 2008, 14:294-296.

38. Eklund KK, Joensuu H: Treatment of rheumatoid arthritis with imatinib mesylate: clinical improvement in three refractory cases. Ann Med 2003, 35:362-367.

39. Ohlsson K, Bjork P, Bergenfeldt M, Hageman R, Thompson RC: Interleukin-1 receptor antagonist reduces mortality from endotoxin shock. Nature 1990, 348:550-552

40. Xing Z, Gauldie J, Cox G, Baumann H, Jordana M, Lei XF, Achong MK: IL-6 is an antiinflammatory cytokine required for controlling local or systemic acute inflammatory responses. J Clin Invest 1998, 101:311-320. 
41. Giebelen IA, van Westerloo DJ, LaRosa GJ, de Vos AF, van der Poll T: Local stimulation of alpha7 cholinergic receptors inhibits LPS-induced TNFalpha release in the mouse lung. Shock 2007, 28:700-703.

42. Chen Z, Zhang X, Chu X, Song K, Jiang Y, Yu L, Deng X: Preventive effects of valnemulin on lipopolysaccharide-induced acute lung injury in mice. Inflammation 2010, 33:306-314.

43. Shi JR, Mao LG, Jiang RA, Qian Y, Tang HF, Chen JQ: Monoammonium glycyrrhizinate inhibited the inflammation of LPS-induced acute lung injury in mice. Int Immunopharmacol 2010, 10:1235-1241.

44. Bonner JC: Regulation of PDGF and its receptors in fibrotic diseases. Cytokine Growth Factor Rev 2004, 15:255-273.

doi:10.1186/cc12786

Cite this article as: Kim et al:: Effect of tyrosine kinase inhibitors,

imatinib and nilotinib, in murine lipopolysaccharide-induced acute lung injury during neutropenia recovery. Critical Care 2013 17:R114.

\section{Submit your next manuscript to BioMed Central} and take full advantage of:

- Convenient online submission

- Thorough peer review

- No space constraints or color figure charges

- Immediate publication on acceptance

- Inclusion in PubMed, CAS, Scopus and Google Scholar

- Research which is freely available for redistribution

Submit your manuscript at www.biomedcentral.com/submit 